\title{
Cinco Proyectos de Innovación Educativa para el diseño y desarrollo de un modelo lexicográfico y de una metodología de aprendizaje de lenguas declinables en estadios iniciales: su aplicación al latín.
}

Manuel Márquez Cruz ${ }^{\mathrm{a}}$ y Ana María Fernández-Pampillón Cesteros ${ }^{\mathrm{b}}$

aUniversidad Complutense de Madrid, manmarqu@ucm.es, bUniversidad Complutense de Madrid, apampi@filol.ucm.es.

\section{\$EWWDWW}

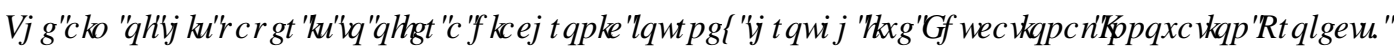

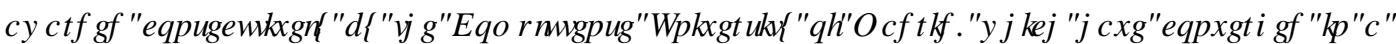

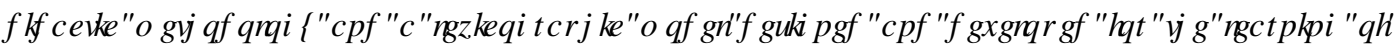

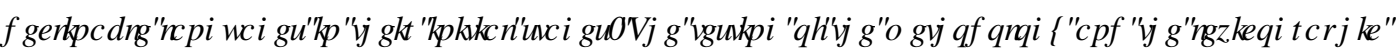

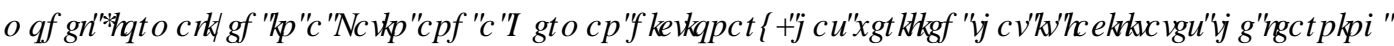

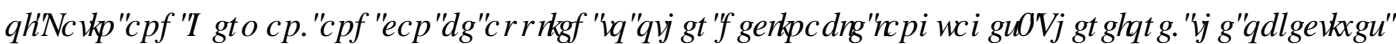

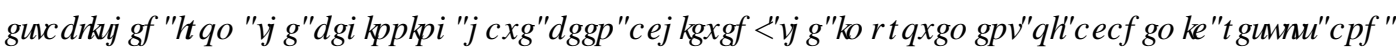

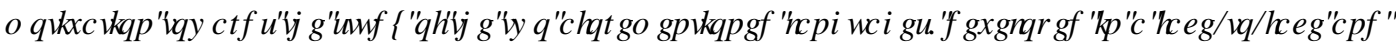

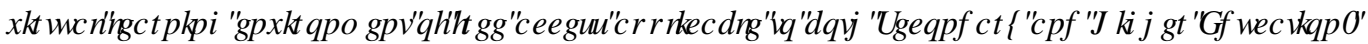

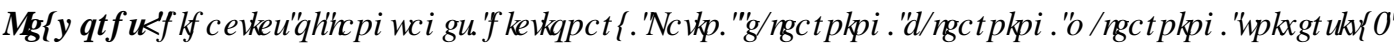

\section{HXP HQ}

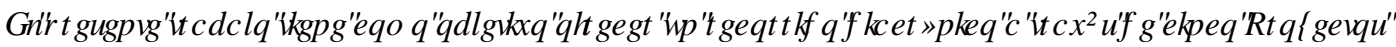

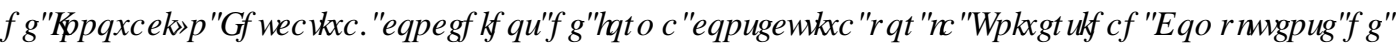

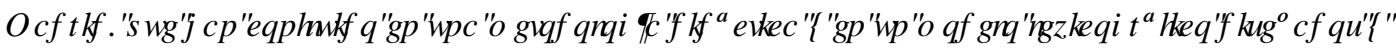

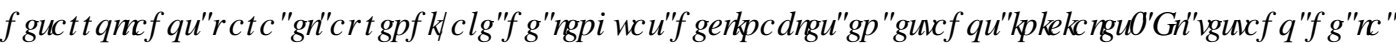

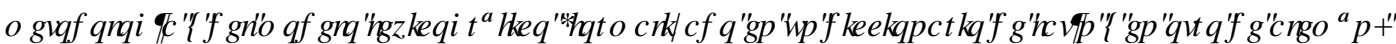

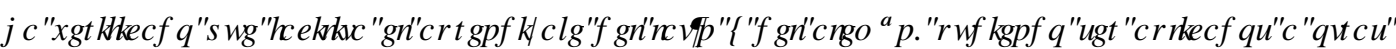

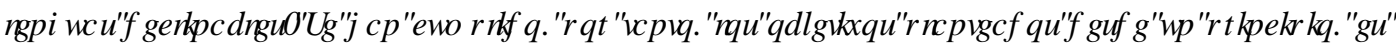

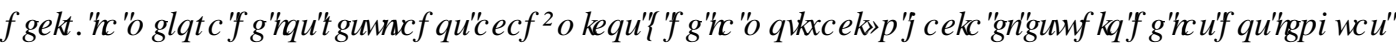

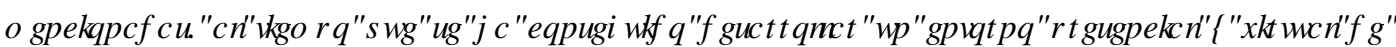

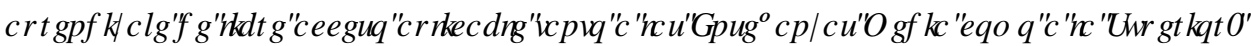

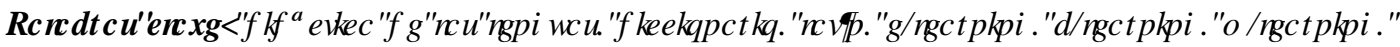
ХQІYНИGDGD

\section{Introducción}

Hace cinco años López de Lerma y Ambrós (2016) publicaron un artículo en el que argumentaron la imperiosa necesidad de satisfacer la demanda de innovación metodológica en la enseñanza del latín, una necesidad de la que ya se había hecho eco un número importante de docentes, según se constata en un estudio previo ${ }^{1}$. Dicho estudio ponía de manifiesto la preocupación del profesorado de latín en relación con

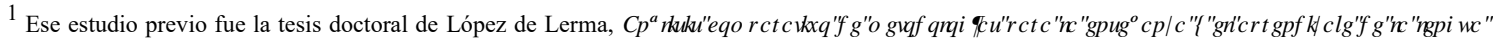
GWQD defendida en la Universitat de Barcelona en el año 2015. Dicha tesis supone un estudio pormenorizado, 186 profesores de 17 comunidades autónomas durante el año 2013
} 


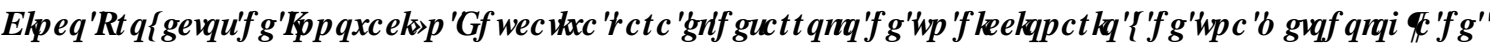

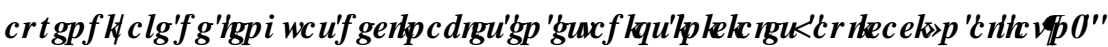

los resultados negativos que estaban deparando las estrategias didácticas y las metodologías empleadas en las aulas de Enseñanzas Medias hasta la fecha. Todo ello traducido en unos resultados académicos que precisaban ser mejorables, así como en una evidente falta de motivación hacia el estudio de la lengua latina. Esta carencia no solo se acusaba y se acusa en las etapas de ESO y Bachillerato, sino en el propio seno de la Universidad, donde existe desde hace unos años un manifiesto interés por mejorar la metodología didáctica del latín, especialmente en los niveles de iniciación. Una clara prueba de ello son los proyectos CILCr (Universidad Complutense de Madrid) y Sal Musarum (Universidad de Cádiz), iniciativas que surgieron como proyectos de innovación educativa para facilitar el aprendizaje en el Curso Cero de Latín, de la UCM, y la inmersión en latín mediante prácticas orales y escritas en el ámbito universitario. No obstante, dichos proyectos universitarios quedaron paralizados, dejando un denotado vacío. La situación de la docencia del latín en la Enseñanza Superior se hizo más acuciante, si cabe, con la implantación del Espacio Europeo de Enseñanza Superior que, tal y como denunciaba el profesor Cristóbal Macías (2012), supuso un nuevo escenario poco alentador para la docencia de la lengua latina. Así pues, en ciertas universidades, como es el caso de la Universidad de Málaga, donde ejerce el autor, se limitó el periodo de docencia de la asignatura Latín a apenas un cuatrimestre en algunas "Carreras", como es el caso del Grado Hispánicas y el de Historia. Hoy, en 2021, sumidos en medio de la pandemia provocada por la COVID-19, la realidad es que ahora, más que nunca, se torna fundamental poner en marcha nuevas metodologías e instrumentos didácticos que además de mejorar los resultados académicos y la motivación en el proceso de aprendizaje-enseñanza, permitan una forma de estudio en la que se combinen entornos presenciales y virtuales.

Con la evidencia de este trasfondo que proporcionaban los citados estudios y su constatación por medio del marco empírico que proporcionaron los datos obtenidos en un Centro docente del Distrito de Salamanca de la ciudad de Madrid, en relación con los resultados académicos que venían obtenidos los alumnos que cursaban la asignatura de Latín en $4^{\circ}$ de ESO, en el año 2016 se solicitó un Proyecto de Innovación Educativa en el Área de Artes y Humanidades de la Universidad Complutense de Madrid, con el objetivo de diseñar, desarrollar, experimentar y valorar una metodología y un modelo lexicográfico didáctico digital inédito que facilitase el aprendizaje de la lengua latina en estados iniciales, aplicable tanto a Enseñanzas Medias como a la Enseñanza Superior. Se buscaba, además, que el modelo didáctico diseñado pudiera ser aplicado al aprendizaje de otras lenguas. A ese primer proyecto le han ido sucediendo otros cuatro proyecto más (convocatorias 2017, 2028, 2019 y 2020) que le han permitido a los IP: a) diseñar y testar una metodología didáctica enfocada a favorecer la iniciación al aprendizaje de lenguas declinables bajo la modalidad e-learning, m-learning y b-learning; b) diseñar y poner en funcionamiento una herramienta lexicográfica digital que facilita el aprendizaje de lenguas declinables; c) crear una red de centros educativos que aplican la metodología y hacen uso del diccionario; d) formar un equipo estable de investigación que comprende especialistas en áreas tan diversas como la docencia del latín y el alemán en diferentes etapas, la enseñanza y el aprendizaje en entornos virtuales, la tecnología educativa, la lexicografía, el análisis de datos y la usabilidad y accesibilidad de los entornos virtuales. Tras cinco años de testado y validación de los modelos diseñados, aplicados en primer lugar a la lengua latina y luego al alemán, la metodología didáctica resultante y el modelo de diccionario diseñado han demostrado su eficacia didáctica, toda vez que se ha podido constatar que facilitan el proceso de aprendizaje-enseñanza de las mencionadas lenguas pudiendo ser aplicados al estudio de otras lenguas declinables.

\section{Objetivos}

El presente trabajo describe desde una perspectiva diacrónica las diferentes fases que han concluido en el desarrollo de un nuevo modelo lexicográfico pedagógico y una metodología didáctica de aprendizaje de lenguas innovadora, aplicable en niveles de iniciación al estudio de lenguas declinables. Cada fase se 
corresponde con uno de los siguientes proyectos de innovación educativa: PIMCD 193:2016, PIMCD 269:2017, PIMCD 164:2018, PIMCD 245:2019 y PIMCD 219: 2020. La concesión de dichos proyectos ha proporcionado un marco de apoyo institucional, financiero y de recursos, que ha confluido en el desarrollo de un espacio virtual de aprendizaje de lenguas declinables abierto y de libre acceso ${ }^{2}$. El presente trabajo se estructura de la siguiente manera:se van a describir cuáles han sido los antecedentes teóricos y prácticos que permitieron la puesta en marcha de los proyectos y cuál sido el marco teórico utilizado para fundamentarlos. A continuación, se va explicar cada uno de los proyectos, en atención a los objetivos planteados y conseguidos, así como a la metodología diseñada. Acto seguido, se van a analizar los resultados, en atención a la transferencia de conocimiento y el impacto de los proyectos. Finalmente, se van a recoger a modo de conclusión los principales logros, así como las líneas de trabajo futuro.

\section{Desarrollo de la innovación}

\subsection{Estado de la cuestión}

En los últimos veinte años, aprovechando el boom de las TIC, se han dado a conocer proyectos de innovación educativa, recursos y metodologías didácticas enfocadas a renovar y actualizar la metodología de enseñanza del latín en nuestro país, anclada hasta no hace mucho en el tradicional método conocido como Gramática-Traducción, enfocado al aprendizaje de reglas gramaticales y de vocabulario para aplicar dichos conocimientos a la decodificación de oraciones o textos. A pesar de que se ha buscado en el uso de las TIC -presentes en todos esos proyectos, recursos y metodologías- fomentar el estudio del latín acercando el aprendizaje de esta lengua a un entorno con el que las nuevas promociones de aprendientes se sienten apatentemente más identificados, tanto los métodos como los recursos diseñados no han llegado a calar con la profundidad que se esperaba, como se muestra a continuación.

Existen varios recursos didácticos en línea que han fomentado el estudio de la lengua latina en atención a aspectos puntuales como son el aprendizaje del léxico, de las declinaciones, de las conjugaciones, de la derivación y de la composición nominal o de la etimología, entre los que destacan las actividades y recursos propuestos por el portal culturaclasica.com ${ }^{3}$. En dicho portal se ofrece una ingente y variada cantidad de actividades para estudiar aspectos gramaticales, morfológicos, sintácticos, etc. propios de la lengua latina. Es más, se proporciona acceso a diferentes números de la revista 3DOHMWD/ DUQD, así como un enlace al FXURV LQ YD/ DUQDSHUHICYWDW, del profesor Ørbeg ${ }^{4}$.

Junto a estos recursos didácticos, se han puesto en marcha, con mayor o menor éxito, diferentes proyectos que han tenido como finalidad proporcionar nuevos escenarios para la enseñanza del latín:

- Palladium ${ }^{5}$ : su objetivo es ofrecer a los docentes un banco de ejercicios, textos e instrumentos didácticos que permiten crear ejercicios autoevaluables. Presenta como principales actividades ejercicios de traducción y análisis de textos con especial atención a aspectos gramaticales como el uso de las formas no personales del verbo.

- $\quad \&, / \& U($ Curso de Introducción a la Lengua y la Cultura Romana): coordinado por J.J. Caerols Pérez, profesor del Departamento de Filología Latina (ahora Clásica) de la Universidad Complutense de Madrid, concluyó en la confección de una herramienta didáctica que sirvió de apoyo durante varios años a la docencia del Curso Cero de Latín, impartido en la misma Universidad. Debido a problemas tecnológicos, la herramienta lleva varios cursos académicos sin utilizarse. Se desarrolló con la finalidad de dotar a los estudiantes universitarios que se iniciaban

\footnotetext{
${ }^{2}$ Los nombres de los profesores, investigadores y estudiantes que han formado parte de estos proyectos de innovación educativa se recogen en el apartado Equipos de trabajo de los diccionarios de latín y alemán, cuyas url se especifican en el acápite en el presente trabajo.

3 http://www.culturaclasica.com/lingualatina/index.htm [Consulta: 25 de marzo de 2021]

4 Para actividades más concretas sobre morfología, sintaxis y vocabulario, en la web de la SELat hay un apartado dedicado a la teleformación mediante diferentes recursos online (Teleformación (selat.org)) [Consulta: 25 de marzo de 2021].

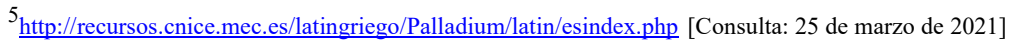

(c)) BY-NC-ND 2021, Universitat Politècnica de València 


\section{\&IQFR]3 UR HFWRGH, QQRVDFYQ( GXFDUDDSDWHDGHDURCR GHXQIGFFRQDURI [GHXQDP HRGRQJTD

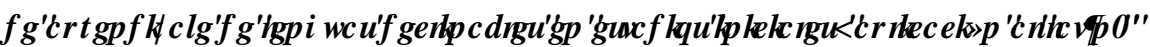

en el estudio del latín de un instrumento didáctico que les guiara por los entresijos de la lengua latina y su cultura. El tiempo de aprendizaje y la consecución de objetivos dependía exclusivamente de la dedicación que se marcara el alumno. Se buscaba, por tanto, que alumnos con niveles de iniciación dispares pudieran convivir en el mismo aula, sin necesidad de restringir los contenidos didácticos ni de que el alumno que tenga un ritmo de aprendizaje más pausado tenga que hacer un esfuerzo extra para alcanzar el nivel de quienes ya tienen cierta base.

- 6DD 0 XVDXP $\square D G L X X H Q H \square$ WXGRVRV. coordinado por S.I. Ramos Maldonado, profesora del Departamento de Filología Clásica de la Universidad de Cádiz, promueve un aprendizaje del latín siguiendo el modelo didáctico humanista, concediendo una gran importancia al elemento de la oralidad y a la comprensión textual. Si bien es cierto que fuentes de la Universidad de Cádiz nos han confirmado que el proyecto sigue vigente, no hemos encontrado, sin embargo, referencias bibliográficas actuales que nos permitan constatar dicha afirmación.

En ambos casos, como se puede observar, los esfuerzos se han destinado a mejorar el proceso de aprendizaje del latín fundamentalmente en aprendientes universitarios.

Sin embargo, a tenor del estudio de López de Lerma, nuestro proyecto ubicó el punto de partida de las nuevas propuestas metodológicas más atrás, concretamente en el momento en el que los alumnos de Enseñanzas Medias se enfrentan por primera vez al estudio de la lengua latina, a fin de solucionar problemas como la falta de motivación hacia el estudio del latín y la mejora de los resultados académicos. Se consideró que si la metodología resultaba provechosa en esta etapa, se podría aplicar a otras etapas posteriores.

En lo que se refiere a las metodologías de enseñanza de la lengua latina, el abanico de posbilidades no es muy amplio:

- El método Gramática-Traducción: fundamentado en el análisis de reglas gramaticales y excepciones que proporcionan un tipo de conocimiento que luego se aplica a la traducción de oraciones. El aprendizaje del vocabulario por medio de largas listas de palabras es uno de los pilares de esta metodología. Ha sido el método seguido tradicionalmente en la enseñanza del latín. Método Ørbeg (editado en forma de manual titulado / IQJ YDDUQDSHUUHICWWDWD propone un método de aprendizaje de latín de corte inductivo, se ha popularizado en el proceso de enseñanzaaprendizaje del latín como alternativa al tradicional. No obstante, tal y como argumenta el profesor C. Macías Villalobos ${ }^{6}$, el número de horas lectivas es un factor fundamental a la hora de poner en marcha un método que apuesta por conceder una especial relevancia al desarrollo de las competencia lectora y oral para aprender latín. La experiencia universitaria señala, según el profesor Macías Villalobos, esta falta de tiempo entre los factores negativos, por lo que al final se decidió una puesta en marcha metodológica que combinase este método inductivo con el tradicional de Gramática-Traducción.

- Según se recoge en el estudio de López de Lerma, un porcentaje no muy alto de profesores ha puesto en marcha una metodología híbrida que consiste en la mezcla de ambos métodos (el tradicional, esto es, Gramática-Traducción, y un acercamiento al aprendizaje inductivo). Pero los resultados no arrojan datos alentadores, en cuanto a la mejora de la motivación hacia el estudio del latín ni al de los resultados académicos (López de Lerna, 2015). Macías Villalobos junto con otros miembros del Proyecto de Innovación Educativa PIE009-2013 decidió aplicar una metodología didáctica de corte inductiva, en la que el aprendizaje del latín partía de la lectura y la comprensión de dicha lengua, a partir de un uso activa de la misma. Los materiales creados siguen aún vigentes y utilizados en la Universidad de Málaga.

Como se puede observar, el panorama didáctico de la enseñanza del latín en estados iniciales dentro del territorio español se reduce, fundamentalmente y según las investigaciones recientes, a la puesta en marcha de una metodología tradicional, la Gramática-Traducción, y a otra de corte inductiva, el conocido como método Ørbeg.Y entre media, aplicaciones híbridas de ambos métodos.

\footnotetext{
6 Macías Villalobos, C. (2012 y 2015), “Algunas consideraciones y materiales para abordar la enseñanza del latín según una metodología híbrida”, Thamyris 6 (2015) 201-300; Macías Villalobos, C. (2012), "La aplicación del método inductivo-contextual a la enseñanza del latín en el ámbito universitario: una experiencia", Thamyris 3 (2012) 151-228.
} 


\subsection{Marco teórico de los proyectos}

El planteamiento inicial de la metodología didáctica surge del uso de un instrumento lexicográfico cuya confección pretende clarificar a los usuarios patrones de comportamiento morfo-semántico-sintáctico de los verbos latinos. Para proceder a tales efectos, se ha diseñado una serie de actividades cuya resolución mediante el uso del diccionario facilita un aprendizaje de tipo inductivo. Todos los materiales didácticos se encuentran alojados en uno de los repositorios de libre acceso de la Universidad Complutense de Madrid. Para el diseño de la metodología didáctica y del propio del diccionario se siguieron los principios lingüísticos de la Gramática Dependencial de L. Tesnière (1959) ${ }^{7}$, aplicados a un tipo de descripción lexicográfica fundamentada en la teoría de los marcos predicativos de Dik (1978 y 1997): se ha definido el concepto de valencia verbal, distinguiendo entre valencia cuantitativa (la especifica el número de complementos obligatorios que requiere el verbo) y valencia cualitativa (la descripción morfológica y ontológica de los complementos obligatorios del verbo), así como los conceptos de argumento (complementos obligatorios) y satélite (complementos facultativos). El diseño permite, además, especificar la función semántica de los argumentos verbales, según la clasificación de dichas funciones propuesta por Dik, fundamentada a su vez en la teoría de casos de Fillmore (1968). Esta teoría se ha utilizado tanto en las explicaciones teóricas (lecciones) como en la confección de la microestructura del diccionario diseñado. El uso del concepto de valencia verbal le permite al alumno identificar el número y la forma de los complementos obligatorios de un verbo, en atención a su significado. Por su parte, en la descripción ontológica de los argumentos se utilizó una adaptación de las entidades de primer y segundo orden de Lyons (1977), utilizada por Márquez Cruz y Chaves Yuste (2016), distinguiendo entre unidades léxicas +animado +humano (seres humanos), +animado -humano (animales y plantas), -animado +definido (objetos y entidades tangibles), -animado -definido (conceptos, sentimientos, entidades abstractas) y lugar.

En relación con la estructura del diccionario, su diseño atiende a los elementos constitutivos y facultativos de un diccionario, según la teoría de la forma del diccionario (Wiegand y Fuentes Morán, 2010): la hiperestructura del diccionario se confeccionó teniendo en cuenta los rasgos inherentes a un diccionario de aprendizaje de lengua (Heuberger, 2018): definiciones, ejemplos, información gramatical y de uso, accesibilidad de los datos, prólogo y apéndice. Se ha prescindido de la información referente a las colocaciones, pronunciación ${ }^{8}$, información de palabras frecuentes y etimología, por no ser rasgos acordes a los objetivos del diccionario de latín.

En cuanto al repositorio donde se alojaron los materiales didácticos, se utilizó el software $\operatorname{OdA}^{9}$ : se trata de un contenedor que permite almacenar, gestionar y publicar colecciones de objetos digitales. La información se organiza según en datos (atributos y valores), recursos (archivos relacionados con el objeto digital) y metadatos (atributos y valores que documentan y clasifican los objetos digitales). El software ha sido creado por el Grupo de investigación ILSA ${ }^{10}$.

\subsection{Metodología: cinco Proyectos de Innovación Educativa para el desarrollo de una herramienta lexicográfica y una metodología didáctica de aprendizaje de lenguas declinables}

La consecución de los objetivos planteados en cada uno de los cinco proyectos ha sido posible gracias a la aplicación de un método de investigación empírico orientado a la validación de los resultados de investigación sobre escenarios y desarrollos reales, con el fin de mejorar los desarrollos (Avison, D, Lan, F, Myers, M, y Nielsen, A., 1999). Por otro lado, la experiencia e idoneidad del equipo investigador

\footnotetext{
${ }^{7}$ Un estudio preciso y conciso de esta teoría se encuentra en Ágel, - Fischer, K (2015).

${ }^{8}$ El Diccionario Didáctico Digital de Alemán sí contempla este rasgo.

9 Fernández-Valmayor Crespo, Alfredo y Fernández-Pampillón Cesteros, Ana María y Varadero Software Factory, VSF (2013) Guía de Gestión del

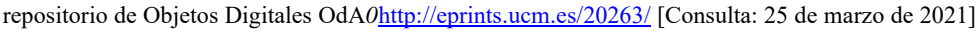

${ }^{10}$ Agradecemos al grupo de investigación Ingeniería de Lenguajes Software y Aplicaciones su soporte tecnológico e inestimable colaboración.
} 


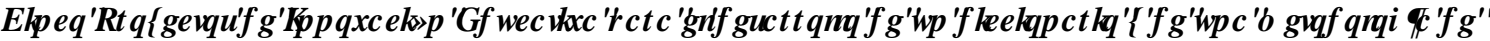 DSUHQ] DNFIGHODJXDVG FCQDEONHQHHDGRVIQIFIDOMUDSCFDFYQDOOWQW}

multidisciplinar ha sido clave en el desarrollo de la metodología didáctica y de la confección de los diccionarios resultantes (latín y alemán). El último de los proyectos cuenta con 20 investigadores, lo que supone un fortalecimiento de sus diferentes áreas: docencia del latín y del alemán, enseñanza y aprendizaje en entornos virtuales, Lexicografía, accesibilidad y usabilidad de datos, métodos de experimentación y tecnologías educativas.

Describimos, a continuación, cada uno de los proyectos, en atención a objetivos y metodología.

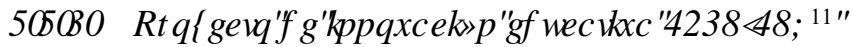 \\ 2 ENAMIRV}

Este primer proyecto tuvo como RENAMKRWJHQHDOAMla creación de una versión digital en línea y en abierto de un modelo de diccionario inédito y el estudio de su viabilidad como herramienta de aprendizaje de lenguas declinables. Como RENAMRRWHSHFIIFRV se determinaron (i) el diseño de una macroestructura del diccionario didáctico digital apropiada para un aprendizaje presencial de lenguas y una autoformación en línea, (ii) la implementación del diccionario didáctico digital para el latín, el uso y la evaluación experimental de la eficacia didáctica del diccionario digital en un aula presencial, (iii) la evaluación teórica de la calidad educativa digital conforme al borrador de norma de AENOR5 y (iv) el estudio preliminar de su viabilidad para otras lenguas derivadas del latín -español- y no derivadas del latín -inglés, alemán o ruso.

Tanto los objetivos generales como los específicos se cumplieron en su totalidad, materializados en dos obras lexicográficas diseñadas para el aprendizaje del latín y del alemán en estadios iniciales, el ' IFFIRQDUR]

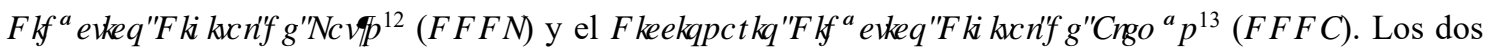
diccionarios se alojaron en los repositorios de la Universidad Complutense de Madrid, mediante el repositorio de objetos OdA, facilitanto su libre acceso. Los diccionarios se diseñaron conforme a los estándares lexicográficos de aprendizaje de lenguas (Heuberguer, 2018) ya citados. En cuanto a la consulta de los datos, uno de los constituyentes obligatorios que determina la forma del diccionario ${ }^{14}$, se puede realizar mediante tres vías: búsqueda por palabra, búsqueda por formulario y navegación a través de la categoría gramatical.

La herramienta fue experimentada y testada en un aula de $4^{\circ}$ de ESO de latín para comprobar su efectividad. El análisis de la experimentación arrojó resultados positivos en cuanto a la mejora del rendimiento académico de los alumnos, que realizaron un test inicial, previo al uso del diccionario, y un test final, posterior al uso del mismo. El resultado de la evaluación indicó la necesidad de llevar a cabo ciertas acciones de mejora que afectaban a la hiperestructura, macroestructura, microestructura e iconoestructura del diccionario (formato, diseño, inclusión de actividades, instrucciones de manejo, imágenes y nuevos términos), efectuadas en los proyectos sucesivos.

\section{HRGRQRJTD}

La metodología seguida durante este primer proyecto se estructuró en las siguientes fases:

1. Definición de la estructura del diccionario digital.

2. Construcción de la primera versión del diccionario conforme a la estructura definida en 1. Se utilizó la herramienta software OdA y las entradas en papel ya probadas en experimentos previos a este proyecto $^{15}$.

3. Evaluación empírica mediante el primer caso de estudio.

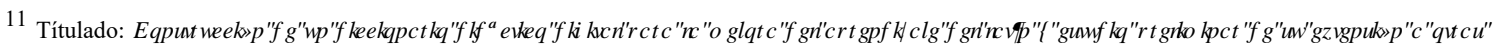
ODIXDV

12 Diccionario Didáctico de Latín (ucm.es) [Consulta: 25 de marzo de 2021]

13 Diccionario Didáctico de Alemán (ucm.es) [Consulta: 25 de marzo de 2021]

14 Sobre la Teoría de la Forma del diccionario y los constituyentes obligatorios y facultativos de los diccionarios, vid. Wiegand- Fuentes Morán (2010).

15 http://eprints.ucm.es/30955/[Consulta: 25 de marzo de 2021]
}

(c)) BY-NC-ND 2021, Universitat Politècnica de València

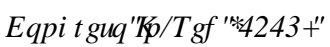


4. Corrección y ajuste del diccionario conforme a los resultados de la evaluación empírica.

5. Experimentación empírica en el aula mediante el segundo caso de estudio (con estudiantes de ESO).

6. Evaluación de resultados durante la experimentación.

7. Evaluación de resultados al final de la experimentación.

8. Incorporación de nuevas entradas del diccionario hasta un total de 693 entradas en el diccionario de latín y 118 en el diccionario de alemán.

9. Evaluación de la calidad educativa del diccionario conforme a la norma UNE 71632.

La estructura del diccionario, que sirvió para confeccionar el ' ' ' / y el ' ' ' \$, se diseñó a partir de un modelo inicial en papel, cuyos fundamentos teóricos, utilidad didáctica y evaluación de los resultados académicos han sido publicados en diferentes trabajos (Márquez Cruz y Chaves Yuste, 2016; Márquez Cruz, 2016).

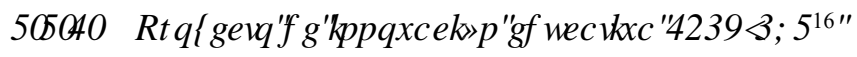 \\ 2 ENAMYRV}

El segundo proyecto se planteó con el RENAMHRIJHQHDOde aplicar y de evaluar empíricamente la efícacia didáctica de la metodología de aprendizaje de lenguas previamente diseñada, para lo cual, se consideró necesario integrar el diccionario en un entorno que facilitase el aprendizaje en escenarios de tipo b-learning y m-learning. En cuanto a los RENAMKRVHHSHFIIFRV, se propuso (i) el diseño de un plan didáctico que debía comprender actividades autoformativas para los escenarios señalados, aplicados al aprendizaje en estados iniciales de la lengua latina y del alemán. En dicho plan, el diccionario tenía que integrarse como una herramienta lingüística de consulta obligada. Se contemplaron también como objetivos específicos (ii) la necesidad de elaborar un informe de evaluación de la calidad didáctica (utilizando la herramienta de evaluación de la calidad de programas e-learning ECB Check $^{17}$ ) y (iii) la evaluación empírica de la eficacia didáctica de la metodología y del diccionario, tomando como escenario de actuación los niveles de Enseñanzas Medias y Enseñanza Superior, en relación al aprendizaje de las dos lenguas sometidas estudio.

El método que se experimentó y validó partió de la hipótesis de que un alumno empoderado supera con mayor facilidad las dificultades que puede plantear el aprendizaje de una nueva lengua. Para conseguir el empoderamiento, se partió de la idea de que el aprendiente debía utilizar los conocimientos de comportamiento semántico-morfológico de los verbos de su lengua materna con el fin de entender el comportamiento de esos mismos verbos en la lengua objeto de estudio. Los esfuerzos debían enfocarse al aprendizaje de los principios básicos de morfología de la lengua L2 y de su sintaxis, entendida esta última como la relación entre el significado de un verbo y el número de sus complementos verbales obligatorios, su roles semánticos y su morfología. Como habrá notado el lector, se evitó el aprendizaje de sintaxis basado en etiquetas que marcan funciones sintácticas (sujeto, complemento directo, complemento indirecto, etc.), dado que dicha información lastra el aprendizaje, tanto en el caso de aquellos alumnos que presentan carencias cognitivas de base que afectan al reconocimiento de dichas funciones en el estudio de su lengua materna, como en el de la falta de correspondencia entre la función sintáctica de ciertos argumentos de los verbos latinos con respecto a su supuesto equivalente en en el español ${ }^{18}$. Para evitar que las definiciones de los diccionarios se conviertan en meras equivalencias léxicas entre dos lenguas, se optó por utilizar la técnica definitoria de parafrasear el significado del verbo lematizado, enumerando los argumentos que intervienen en el evento descrito: en el caso del verbo latino $G$, se define como "alguien le da algo a alguien". Aplicado este conocimiento a la estructura básica de la oración latina -puesto que se parte siempre

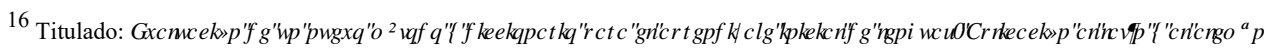

${ }^{17}$ http://www.ecb-check.net// [Consulta: 25 de marzo de 2021]

${ }^{18}$ Sirvan como ejemplo el verbo GRFR, que en latín rige dos acusativos, mientras que en su traducción al español rige un CD y un CI: "enseñar gramática a los estudiantes".
}

(c) B BY-NC-ND 2021, Universitat Politècnica de València

CRQJHMR, Q5HGHपिए। 


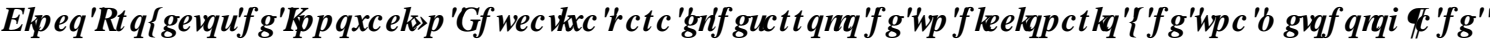

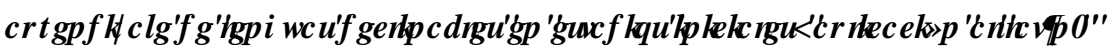

de ejemplos oracionales- se explica dicha oración a modo de rompecabezas, en el que la pieza clave y central es el verbo, que necesita, según su significado, un número fijo de argumentos (complementos obligatorios), esto es, el resto de piezas del puzle necesarias para que el significado de la oración sea adecuado, coherente y medie la cohesión.

El proyecto concluyó con la consecución de los objetivos planteados: se diseñó un plan formativo en el que el diccionario implementado se convirtió en el instrumento de uso en torno al cual gira toda la metodología diseñada de aprendizaje de lenguas. Se construyó una herramienta web interactiva de aprendizaje de lenguas declinables (una página web estática que contiene un curso Moodle) y se evaluó su calidad ${ }^{19}$ y eficacia didáctica contrastando los resultados obtenidos en diferentes pruebas con los del uso de una metodología tradicional, como es el caso de la Gramática-Traducción. Se comprobó que la herramienta implementada permitía en este primer estadio un aprendizaje en formato e-learning y b-learning: la interfaz del diccionario proporciona el acceso a unos videos formativos y a unas lecciones que plantean unos ejercicios a resolver mediante el uso del diccionario.

Para proceder a tales efectos, fue necesario el diseño de un itinerario didáctico que proporcionó una estructura general, que solo había que rellenar con los contenidos de las diferentes unidades didácticas, en atención a la lengua de aprendizaje. No obstante, la falta inicial de un método sistemático que agilizara y rentabilizara el proceso de grabación de los videos didácticos supuso un retraso en la implementación de estos materiales. Ahora bien, aunque no se desarrollaron todas las lecciones se diseñó y puso en marcha un método propio de creación de materiales que rentabilizó los resultados de los siguientes proyectos, como demuestran los prototipos de lecciones publicadas en el ' ' ' / y el ' ' ' \$. Se adquirió, por tanto, suficiente experiencia para poder terminar la implementación del recorrido didáctico en el siguiente proyecto.

\section{HRCRQRTD}

El proyecto se desarrolló aplicando una metodología basada en la solicitud y en la metodología Design for Change (en adelante DC), una nueva metodología de desarrollo de proyectos educativos ${ }^{20}$ sobre la que los miembros del proyecto recibieron formación gracias al proyecto de la UCM, INNOVA 196: 2016-17. De forma más detallada el proyecto se organizó en las cinco fases y actividades siguientes:

1. Análisis conjunto del problema, de experiencias y de recursos (fase "Siente" en la metodología DC).

2. Diseño del plan didáctico e-learning y de mejoras para los diccionarios didácticos digitales (fase "Imagina" en la metodología DC).

3. Implementación de los diseños (fase "Actúa" en DC): mejoras en los diccionarios, lecciones e itinerarios de latín y alemán.

4. Evaluación empírica de la eficacia didáctica y corrección de deficiencias (fase "Evalúa" en DC).

5. Difusión de los resultados (fase "Comparte" en DC): evaluación final de los resultados, y redacción de informes.

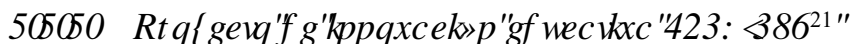

\section{ENAMVRV}

Los itinerarios didácticos diseñados durante el proyecto 193, así como la puesta en funcionamiento del espacio web que permitía el libre acceso a la herramienta lexicográfica, a las lecciones y a las actividades

\footnotetext{
${ }^{19}$ El método de evaluación fue una evaluación por pares utilizando, como herramienta de evaluación, el perfil del profesor de la norma UNE 71362 de calidad de materiales educativos digitales disponibles en línea en http://loep.dit.upm.es/ (es necesario registrarse).

20 http://www.dfcspain.org/ [Consulta: 25 de marzo de 2021]

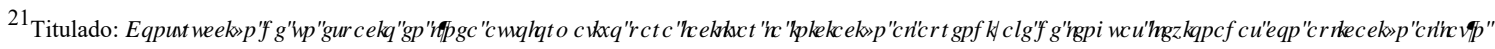
I $\triangle D O P$ iQ
} 
didácticas proporcionaron el contexto adecuado para emprender este nuevo proyecto. Se habían asentado las bases de un sistema de iniciación al aprendizaje de lenguas declinables en espacios presenciales y virtuales para hablantes de español, cuyo componente pedagógico comprendía teorías de aprendizaje, estrategias didácticas y metodologías E-A:

\begin{tabular}{|c|c|c|}
\hline Teorías de aprendizaje & Estrategias didácticas & Metodologías E-A \\
\hline Aprendizaje cognoscitivo & Análisis de casos & E-Learning \\
\hline & $\begin{array}{l}\text { Aprendizaje basado en } \\
\text { equipos }\end{array}$ & B-Learning \\
\hline
\end{tabular}

No obstante, se observó que los resultados podrían mejorar sustancialmente si el espacio virtual se hacía dinámico y las actividades abandonaban la estaticidad en favor de una interactividad. Se determinó, por tanto, como RENAMARUHQHDOtransformar el espacio virtual de aprendizaje de lenguas flexivas y declinables ya construido en un espacio virtual autoformativo, un espacio que le permitiera al aprendiente trabajar a su propio ritmo en atención a aquellas necesidades concretas que demande la fase del proceso de aprendizaje en la que se encuentre. Para conseguir este objetivo, se especificaron como RENAMVRVHSHFIIIFRV(i) la construcción del material educativo digital (videolecciones y ejercicios interactivos, fundamentalmente), (ii) la evaluación de la calidad de los materiales y del espacio virtual, lo cual incluye la evaluación de la accesibilidad universal, (iii) la evaluación empírica de la eficacia didáctica del espacio virtual de aprendizaje para corregir posibles puntos susceptibles de mejora, (iv) la evaluación teórica de la efícacia didáctica del espacio virtual basada en el análisis de los fundamentos cognitivos y neurolingüísticos que subyacen con el fin de ajustar, si fuese necesario, el método o el diccionario respecto a su eficacia didáctica y, por último, (v) la puesta en marcha del espacio de aprendizaje en experiencias educativas reales durante el curso 2018-2019 en los niveles de Enseñanzas Medias y Superior

Los objetivos propuestos, generales y específicos, se alcanzaron con la creación de un espacio virtual de libre acceso y autoformativo para el aprendizaje en estados iniciales del latín y del alemán. Estos espacios, que fueron validados en cuanto a su eficacia didáctica, están disponibles en abierto con licencia creative commons de Reconocimiento-CompartirIgual:

Curso de iniciación al latín: https://cv4.ucm.es/moodle/course/view.php?id=115039

Curso de iniciación al alemán: https://cv4.ucm.es/moodle/enrol/instances.php?id=115038

Dichos enlaces se ubicaron el sitio web de los diccionarios, en la sección "Curso de iniciación al latín" y "Curso de iniciación al alemán".

Se crearon las cuatro primeras lecciones digitales para cada una de las lenguas del proyecto: latín y alemán. Cada lección está formada por una explicación del profesor en formato multimedia (videolección, audiolección y texto), una autoevaluación con autocorrección y una propuesta de ejercicios, también autocorregibles, para practicar. Las lecciones se crearon con el editor de contenidos didácticos eXeLearning, una aplicación de software libre mantenida y distribuida por el INTEF ${ }^{22}$. Se seleccionó esta aplicación porque permite crear los materiales educativos digitales en diferentes formatos estándar (html, scorm e ims-cp), a fin de conseguir portabilidad e independencia de la tecnología en grado máximo. Asimismo, eXeLearning facilita el cumplimiento de los criterios de accesibilidad tecnológica y la creación del ecosistema que permite la producción y actualización sistemática de las lecciones digitales: (i) un

22 http://exelearning.net/[Consulta: 25 de marzo de 2021]

(c) B EY-NC-ND 2021, Universitat Politècnica de València

CRQJUHR, Q5 HGHपिए। 


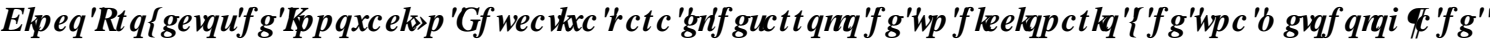

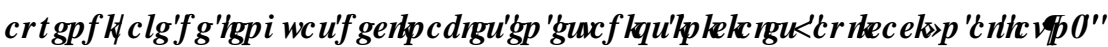

prototipo de lección digital, (ii) un repositorio compartido en Drive, y, (iii) un método de creación de videolecciones que, con un coste mínimo, ayuda a obtener buenos resultados desde el punto de vista didáctico, tecnológico y de accesibilidad. Este método se especifica en una guía publicada en el repositorio de eprints de la $\mathrm{UCM}^{23}$. En cuanto a la evaluación de la calidad del espacio autoformativo, se llevó a cabo siguiendo los criterios de calidad de la norma UNE 71362 Calidad de Materiales Educativos Digitales. Dichos criterios fueron utilizados como guía durante el proceso de creación las lecciones, a fin de cumplir los criterios obligatorios y de excelencia, o, en todo caso, los criterios de cumplimiento obligatorio. Por cuanto respecta a la evaluación de la efectividad didáctica, se testó en el curso de latín ${ }^{24}$ a partir de unos estándares de motivación, adaptados del AMBT de Gardner ${ }^{25}$. El análisis de los resultados (Márquez Cruz y Fernández-Pampillón Cesteros, 2019) demuestra una mejora significativa de la motivación hacia el estudio del latín.

Los resultados de las dos experiencias piloto de los espacios de aprendizaje "iniciación al latín" e “iniciación al alemán” fueron de gran utilidad puesto que permitieron definir y probar el procedimiento de puesta en marcha, así como detectar los puntos fuertes y débiles de la metodología y de sus instrumentos.

Tanto el ' ' ' / como el ' ' ' \$ se presentaron en el congreso internacional de Lexicografía organizado por eLex en Sintra en 2019 (Márquez Cruz, Férnández- Pampillón Cesteros y Sánchez Hernández, 2019).

\section{HRRRRJTD}

El proyecto se llevó a cabo en cuatro fases:

1. Construcción del espacio virtual de aprendizaje (en paralelo para latín y alemán). Se aplicó el diseño didáctico resultados del PIE 193-2017 todavía en ejecución.

2. Puesta en marcha de los espacios y evaluación empírica de su eficacia didáctica.

3. Evaluación teórica de la eficacia didáctica de los espacios virtuales

4. Publicación de resultado: publicación en abierto del espacio virtual y difusión científica de los resultados obtenidos.

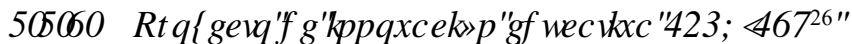 2 ENAMYRV}

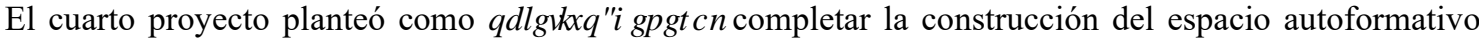
virtual de iniciación al aprendizaje de nuevas lenguas y ponerlo a disposición de cualquier profesor o estudiante, así como darlo a conocer en foros científicos y educativos. Dicho objetivo se concretó en cinco RENAMARVHSHFEIIFRV. (i) la evaluación inicial cualitativa y cuantitativa del espacio virtual, (ii) la corrección o mejora del espacio virtual, una vez analizada la evaluación inicial, (iii) la mejora de los diccionarios en términos cualitativos (ampliación y mejora de la información didáctica) y cuantitativos (incremento del número de lemas), (iv) la implantación del espacio virtual en otros centros educativos (en el contexto de Proyectos de Innovación Educativa, Proyectos Piloto o Convenios de Colaboración) y (v) la difusión de los resultados en foros científicos.

El objetivo general no se pudo cumplir al 100\% como consecuencia de las circunstancias excepcionales derivadas de la pandemia ocasionada por el COVID-19. El cierre de colegios, institutos y universidades el

\footnotetext{
23 https://eprints.ucm.es/56866/ [Consulta: 25 de marzo de 2021]

${ }^{24}$ En el curso de alemán no se pudo llevar a cabo la evaluación de la motivación, debido a que la prueba inicial no fue realizada correctamente por los alumnos (se duplicaron encuestas y hubo dos alumnos que no llegaron a hacer la prueba).

${ }^{25}$ Gardner, R.C. (2004) Attitude/Motivation Test Battery: International AMTB Research Project. London: University of Western Ontario.

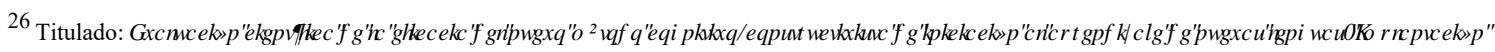

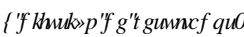


11 de marzo de 2020, supuso la suspensión de la enseñanza presencial en todos los niveles educativos, lo que impidió la completa evaluación-corrección del espacio autoformativo virtual. Solo se pudo llevar a cabo la experimentación en dos centros docentes en el mes de diciembre de 2019, y sólo en el caso del latín. Ahora bien, el análisis de las pruebas efectuadas volvió a demostrar la eficacia de la metodología aplicada y del uso del instrumento didáctico, en términos de motivación hacia el estudio del latín y de resultados académicos, toda vez que la comparativa entre las pruebas iniciales y las pruebas finales (motivación y resultados académicos) reflejó una mejora significativa en los resultados obtenidos. Asimismo, se pudo confeccionar un cronograma de actuación, en el que quedaron detallados el número de sesiones necesarias para una aplicación efectiva y la actividad a desarrollar en cada una de las sesiones, cronograma que sirvió como documento estándar para la aplicación de la prueba en el siguiente proyecto (solicitado y concedido en octubre de 2020). Se hicieron, además, correcciones sobre los modelos de pruebas y el propio espacio virtual autoformativo, según las anotaciones de campo recogidas por los formadores durante la experimentación, así como por los comentarios críticos del alumnado. En cuanto a la implantación-difusión en otros Centros Educativos, en el mes de febrero se acordó con la dirección de otras dos Instituciones Educativas la puesta en marcha de la metodología en los meses de mayo-junio, pero las mencionadas circunstancias impidieron que el acuerdo se materializase. En lo que se refiere a la difusión de los resultados, sí se pudo llevar a cabo esta acción en distintos foros académicos (Márquez Cruz y FernándezPampillón Cesteros, 2020; Márquez Cruz 2020).

\section{HMRRRJTD}

La metodología del proyecto ha comprendido cuatro fases:

1. Evaluación cualitativa y cuantitativa de la metodología Además de aplicar y evaluar la metodología, se diseñó un cronograma de actuación para aplicaciones futuras.

2. Corrección o ajuste del espacio virtual conforme a las conclusiones de la evaluación y mejora de los diccionarios. Se añadieron nuevos artículos lexicográficos y se mejoró la información a nivel de microestructura de las entradas ya confeccionadas (distribución de ejemplos y nuevas formas de definición lexicográfica).

3. Implantación del espacio virtual. Debido al confinamiento obligado como consecuencia a la situación derivada de la pandemia COVID-19, la fase de implantación solo se pudo cumplir parcialmente. Analizado el proceso de aprendizaje con la metodología experimental propuesta para la enseñanza del latín en un IES de la zona noroeste de la Comunidad de Madrid y en un IES de la zona de centro de Madrid capital, se desarrolló la versión final de la experimentación. Se presentó la metodología a la Dirección de un centro educativo de la zona sur de Madrid y a otro centro de la zona central de Madrid capital. Se acordó con dichos centros la puesta en marcha de la metodología en los meses de mayo y junio, tras unas sesiones de formación al profesorado, en el primero de los centros citados.

4. Difusión de los resultados. La metodología experimental aplicada al latín se ha presentado en forma de comunicación al congreso internacional de lexicografía EURALEX 2020. En las Jornada Innova 2019 (celebradas en noviembre de 2020) se expusieron los resultados del presente Proyecto de Innovación Educativa a la comunidad científica que tomó parte en dichas Jornadas

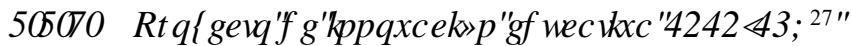 \\ 2 ENAMIRVI}

El RENAHMRIJHQHDOde este último proyecto -vigente aún- es la creación de una red de aplicación, evaluación y difusión de un modelo de espacio autoformativo virtual de iniciación al aprendizaje de lenguas,

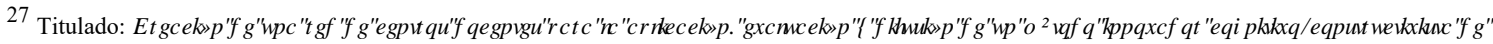

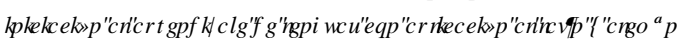

(c)) BY-NC-ND 2021, Universitat Politècnica de València 


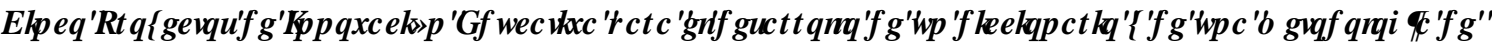

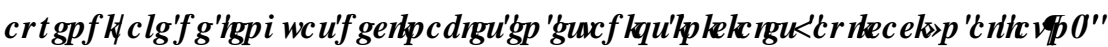

poniéndolo a disposición de diferentes centros-instituciones docentes, profesores y alumnos, así como darlo a conocer en foros científicos y educativos. Este objetivo principal se desglosa en los siguientes RENAMRRV HSHFIIFRs: (i) la creación de una red de centros docentes, representantes de entornos sociales variados, y la aplicación y evaluación del espacio virtual de aprendizaje, (ii) la corrección, mejora o adaptación del espacio virtual, (iii) la actualización de los diccionarios, incluyendo nuevos artículos lexicográficos y mejorando la información didáctica y (iv) la difusión de los resultados en publicaciones o foros científicos y educativos.

El primer objetivo específico se estructura en dos etapas: una primera etapa, llevada a cabo entre los meses de noviembre y diciembre, que consiste en aplicar la metodología en tres centros y una segunda etapa, que se consumará en los meses de mayo-junio, mediante la puesta en marcha de la metodología en diferentes Centros Educativos de la Comunidad de Madrid. Para conseguir este último objetivo, ha sido necesaria una evaluación de la fase primera, a fin de aplicar las correcciones pertinentes sobre la metodología, dado que los escenarios de docencia durante el presente curso académicos han cambiado con respecto a otros años como consecuencia a la COVID-19 (puesta en marcha de aulas virtuales en paralelo a presenciales para clases que superen un número de alumnos que ponga en riesgo las condiciones sanitarias).

En el momento de redactar este trabajo, el proyecto se encuentra al final de la fase dos: se ha aplicado la metodología de aprendizaje de latín en tres Centros Educativos de la Comunidad de Madrid, se han analizado los resultados y se han propuesto acciones de mejora que están a punto de concluir en una nueva versión de la metodología desarrollada. Por otro lado, se están ampliando las entradas lexicográficas de los diccionarios de latín y de alemán, con el objetivo de llegar hasta los mil lemas.

\section{HRGRQRJDD}

El proyecto comprende las siguientes fases:

1. Creación de una primera red de centros docentes: aplicación y evaluación de la metodología.

2. Corrección, mejora o adaptación del espacio virtual y de los diccionarios, aplicando los resultados de la evaluación.

3. Ampliación de la primera red de centros para la aplicación y difusión del espacio virtual autoformativo y de la metodología.

4. Difusión de los resultados: preparación de, al menos, un artículo sobre la aplicación de la metodología y los resultados, y una comunicación en un congreso, donde se explique la metodología.

\section{Resultados}

El recorrido diacrónico por los cinco proyectos demuestra la evolución de una metodología de aprendizaje de lenguas flexivas declinables, que partió de un prototipo analógico, cuyos primeros materiales didácticos fueron unas fichas lexicográficas en papel, hasta llegar a un espacio de autoaprendizaje virtual de libre acceso. Durante estos cinco años, la evaluación de las diferentes pruebas realizadas ha devuelto valores significativos que demuestran que la metodología desarrollada incide en un mejor rendimiento académico y repercute en la motivación hacia el estudio de dos lenguas, como son el latín y el alemán. La continua experimentación y evaluación ha permitido crear una metodología y unos instrumentos de aprendizaje de lenguas que han superado los requisitos de calidad y usabilidad. La herramienta lexicográfica, por su parte, se ha convertido en un prototipo de diccionario didáctico presentado y aceptado en dos congresos internacionales de lexicografía. Su aplicación es apta para tanto para los distintos niveles de enseñanza, en cursos de iniciación al aprendizaje del latín y del alemán, dados los objetivos didácticos propuestos. El análisis estádistico de los datos que prorporcionan las pruebas realizadas durante los sucesivos años 
académicos revelan diferencias estadísticamente significativas, toda vez que la media de la diferencia en el número de errores entre pretest y postest es positiva ${ }^{28}$.

La metáfora del puzle ha sido valorada por los estudiantes como una estrategia solvente que ayuda a entender de una manera sencilla el funcionamiento de la complementación verbal en las dos lenguas aplicadas. Se ha conseguido el empoderamiento del alumnado a la hora de emprender el estudio de una lengua que, por sus características morfológicas, difiere del español. Lejos de rendirse ante la dificultad que supone enfrentarse al aprendizaje de una lengua declinable, el alumno -en un alto porcentaje- se ha sentido cómodo trabajando, ha demostrado asimilar los conocimientos y se ha sentido protagonista de su proceso de aprendizaje. Es especialmente relevante el hecho de aquellos alumnos que presentaban carencias de base en relación con los conocimientos lingüísticos del español obtuvieron buenos resultados académicos y llegaron a comprender el funcionamiento de la complementación en las dos lenguas estudiadas. Bajo la forma de aprendizaje significativo, se ha desarrollado una metodología didáctica integradora, que supone todo un avance en el ámbito de la atención a la diversidad: el método diseñado permite que la sesión sea seguida al mismo tiempo por alumnos que presentan niveles diferentes en cuanto a sus habilidades lingüísticas.

La colaboración entre Colegios, Institutos y Universidad ha sido fundamental para la consecución de los diferentes proyectos, apoyados por otras instituciones como la Sociedad de Estudios Latinos, la Sociedad Española de Estudios Clásicos, la Federación de Asociaciones de Germanistas, el Departamento Filología Latina de la Universidad Complutense de Madrid y la Sección de Alemán y Laboratorio de Ingeniería Didáctica de la Universidad Nacional de Educación a Distancia. Todo ello se ha traducido en una transferencia de conocimiento y en un impacto académico, cuantificado a partir de los siguientes logros:

- Material formativo de eficacia didáctica probada para el aprendizaje de la lengua latina y alemana.

- Patente: Propiedad intelectual del Diccionario Didáctico Digital de Latín

- Publicaciones en revistas científicas.

- Participación en Congresos y Seminarios

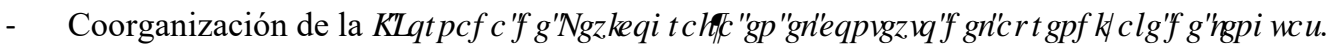

- Organización de talleres de formación del profesorado.

- Firma de convenios de colaboración con centros docentes.

- Confección de diferentes Trabajos de Fin de Grado y Trabajos de Fin de Máster.

Con respecto al ' IFFIRQDUR]' IG FWFRIGHV DUQ ha recibido 837 clics web, $26.7 \mathrm{~K}$ impresiones (web) y 53 páginas con primeras impresiones, lo que implica que aparezca en la primera página de búsquedas de diccionarios didácticos. Cuando se consulta "diccionarios didácticos latín" aparece en primera posición. En alemán no se dispone de datos sobre la posición media porque no se ha dado de alta en la aplicación de analíticas de Google. No obstante, si se consulta “diccionarios didácticos alemán” aparece en primera posición.

\section{Conclusiones}

Si bien es cierto que todavía queda camino por recorrer, la concesión de los cinco proyectos ha supuesto el diseño, la experimentación, la evaluación y la materialización final de (i) una metodología innovadora en el ámbito del aprendizaje de las lenguas declinables que incide en la mejora de los resultados académicos y en la motivación hacia el estudio, (ii) una herramienta de aprendizaje que permite alternan su uso bajo el formato de clase presencial y/o virtual y (iii) un diccionario didáctico que servirá como prototipo para la confección de otros diccionarios didácticos, incluso en otras lenguas. Añadimos, además, que con estos

${ }^{28}$ El análisis de los datos va a ser publicado en un estudio posterior, una vez concluya la experimientación del presenta año académico. 


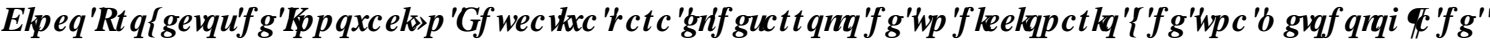

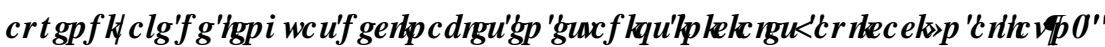

cinco proyectos se ha conseguido (iv) una estrecha colaboración entre el profesorado de diferentes etapas educativas (media y superior), (v) fomentar la investigación en la Universidad por mejorar los procesos de aprendizaje en etapas educativas precedentes, (vi) la consolidación de un grupo de investigación multidisciplinar al que año tras año se van uniendo nuevos miembros y (vii) crear una metodología didáctica del latín y del alemán, apta para su aplicación en la enseñanza universitaria. De hecho, tras varios años de experimentación, corrección y mejora en curso básicos, el análisis de los resultados nos depara una metodología apta para su uso como herramienta didáctica en los Cursos Cero de Latín de las Universidades españolas, así como en las asignaturas de iniciación al latín impartidas en los distintos Grados bajo la nomenclatura de Latín, Latín I o Gramática Latina. Como último objetivo, se diseñó y construyó un espacio didáctico y un diccionario para el aprendizaje del alemán, siguiendo la pauta marcada por el diccionario y la metodología didáctica del latín.

Han permitido, además, que en plena pandemia los docentes pudieran contar instrumentos de apoyo en sus tareas, tanto en entorno presencial como virtual.

La continuación de las acciones acometidas en futuros proyectos supondrá la ampliación de los materiales didácticos, en atención a un nivel superior de aprendizaje, que en concordancia con el marco teórico del proyecto se enfocará al estudio de los papeles semánticos de los argumentos verbales. Dicho estudio facilitará la extrapolación del conocimiento adquirido a otras lenguas no declinables. Al mismo tiempo, se ha de seguir trabajando en la línea de que el usuario pueda seleccionar el tipo de información a la que acceder, en atención a las diferentes necesidades que puedan surgir entre los aprendientes de un mismo aula.

\section{Referencias}

ÁGel, V. y FISCHER, K. (2015). “Dependency Grammar and Valency Theory”, en Heine, B y Narrog, H. (eds.), 7KH2 [IRLCT+DQCERRNRIVIQJ XLMFL\$ QDOUV, Oxford: Oxford University Press, 223-255.

AVISON, D.E., LAU, F., MYERS, M.D. y NIELSEN, P. A. (1999). “Action Research”. \&RP P XQIFDMRQVRIIKH \$\&0 [42, 1, pp. 94-97.

DIK, S.C. (1978). ) XQFWRQDO* UPP P DU North-Holland Linguistic Series, 37, Amsterdam: North-Holland Publishing Company.

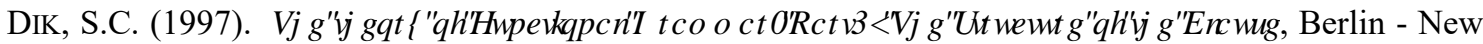
York: Mouton de Gruyter.

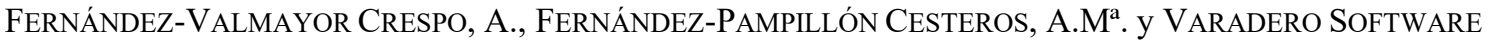

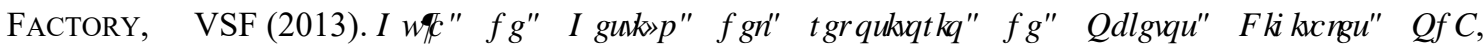
http://eprints.ucm.es/20263/ [Consulta: 25 de marzo de 2021]

FILLMORE, Ch. (1968). "The Case for Case”, en Bach, E. y Robert, T. (eds.), 8 QIYHWDOIQ/ IQJXLMAL 7KHRU, New York: Holt, Rinehart and Winston. 21-119.

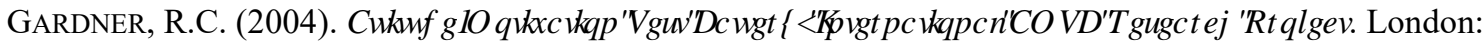
University of Western Ontario.

HEUBERGER, R. (2018). "Dictionaries to assist teaching and learning", en Fuertes-Olivera, P.A. (ed.), 7KH 5RXIAGJH+ DQCERRNRIV HIFRJUSKI, Abingdon - New York: Routledge, pp. 300-316.

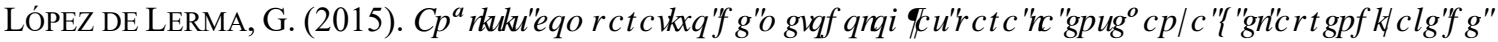

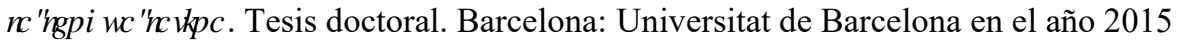


LÓPEZ DE LERMA, G. y AMBRós, A. (2016). "Enseñanza de la lengua latina: resultados preliminares sobre las ventajas e inconvenientes en el empleo de diferentes metodologías", 0 HKRGRV, 3, pp. 67-83.

LYONS, J. (1977). 6HP DQMFV, Cambridge: Cambridge University Press.

MACÍAS ViLlaLOBOS, C. (2015). "Algunas consideraciones y materiales para abordar la enseñanza del latín según una metodología híbrida”, 7KDP \UV6, pp. 201-300.

MaCíAS ViLlalobos, C. (2012). "La aplicación del método inductivo-contextual a la enseñanza del latín en el ámbito universitario: una experiencia”, 7KDP $\backslash U V 3$, pp.151-228.

MÁrquez CruZ, M. (2016). "La Lexicografía al servicio de la docencia: presentación de un modelo de diccionario funcionalista de latín y su aportación al estudio de esta lengua en el aula", en Torrego, A. et al.

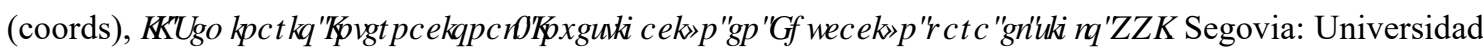
de Valladolid, pp.151-169. http://uvadoc.uva.es/handle/10324/22769 [Consulta: 25 de marzo de 2021].

MÁRquez CruZ, M. y Chaves yuste, B. (2016). “A Latin Functionalist Dictionary as a Self-Learning Language Device: Previous Experiences to Digitalization”, ( GXFDMRQ6FHQFH, 6, 4, pp. 1-19.

MÁrquez Cruz, M. y Fernández-PAMPILlón Cesteros, A.Ma. (2019). "Motivación en el aprendizaje del latín: evaluación de una nueva metodología didáctica”, $5 H$ ' $R \& U H, 8$, pp. 432-441

Márquez Cruz, M., Fernández-Pampillón Cesteros, A.Ma y SÁnchez Hernández, P. (2019). “A novel Cognitive Model of Digital Didactic Dictionary for Learning Foreign Languages. Application to

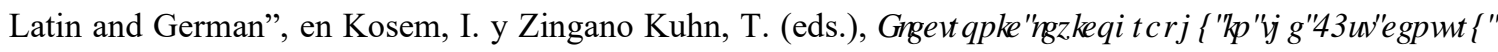

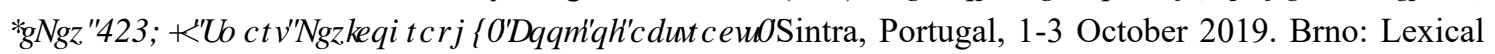
Computing CZ s.r.o. Electronic Lexicography in de 21st century (eLex 2019), pp.40-42.

MÁrquez Cruz, M. y Fernández-PAmpillón, A.Ma . (2020). "A Morpho-Semantic Digital Didactic Dictionary for Learners of Latin at Early Stages", en EURALEX XIX, Lexicography for Inclusion, (EURALEX 2020), 193-202

MÁrquez CruZ, M. (2020). "Evaluación científica de la eficacia del nuevo método cognitivoconstructivista de iniciación al aprendizaje de nuevas lenguas. Implantación y difusión de resultados”, en 7DCHU,1129\$) IROR日u, celebrado el 23 de noviembre de 2020 en la Facultad de Filología de la Universidad Complutense de Madrid. Madrid.

TESNIÈRE, L. (1959). e QP HQWGHV QWD HWUFWWQParis: Librairie C.

WIEGAND, H.E. y FUENTES MORÁN, Ma .T. (2010). ( WUXFWLWV HIFRJU IIFDMIS ISHFRVIFHQWDOMUGHXQD URLDDCH(DI) RLP DCGDI IFFIRQDUR. Granada: Ediciones Traganto. 